\title{
THE ZALCMAN CONJECTURE FOR CLOSE-TO-CONVEX FUNCTIONS
}

\author{
WANCANG MA
}

(Communicated by)

\begin{abstract}
Let $S$ be the class of functions $f(z)=z+\cdots$ analytic and univalent in the unit disk $D$. For $f(z)=z+a_{2} z^{2}+\cdots \in S$, Zalcman conjectured that $\left|a_{n}^{2}-a_{2 n-1}\right| \leq(n-1)^{2}(n=2,3, \ldots)$. This conjecture is verified for $n \geq 4$ and close-to-convex functions.
\end{abstract}

Let $S$ be the class of functions $f(z)$ analytic and one-to-one in the unit disk $D=\{z:|z|<1\}$ and normalization $f(0)=f^{\prime}(0)-1=0$. Recently, Zalcman conjectured that

$$
\left|a_{n}^{2}-a_{2 n-1}\right| \leq(n-1)^{2}
$$

for each $n \geq 2$ and each $f(z)=z+a_{2} z^{2}+\cdots \in S$, which implies the famous Bieberbach conjecture $\left|a_{n}\right| \leq n[\mathbf{1}]$.

It is well known that (1) is valid for $n=2$ [2]. The conjecture is known to be true for the subclass $S_{\mathbf{R}}$ of functions in $S$ with real coefficients. In [1], Brown and Tsao proved that (1) holds for the class $T$ of typically real functions and the class $S^{*}$ of starlike functions. An analytic function $f(z)=z+\cdots$ is typically real if and only if $(\operatorname{Im} z)(\operatorname{Im} f(z)) \geq 0(z \in D)$. And $f(z)=z+\cdots$ is starlike means that $f(D)$ is starlike with respect to the origin.

In this paper, we prove the Zalcman conjecture for the class $C$ of close-to-convex functions and $n \geq 4$. Thus the result in [1] on the starlike functions is an immediate consequence of ours when $n \geq 4$. This provides further evidence in favour of the Zalcman conjecture.

A function $f(z)=z+a_{2} z^{2}+\cdots$ analytic in $D$ is close-to-convex if and only if there exist a real number $\theta$ and a function $g$ in $S^{*}$ such that

$$
\operatorname{Re}\left(e^{i \theta} z f^{\prime}(z) / g(z)\right)>0 \quad(z \in D) .
$$

We denote by $H C$ the closed convex hull of $C$.

Our principal result can now be stated.

THEOREM. Let $f(z)=z+a_{2} z^{2}+a_{3} a^{3}+\cdots \in H C$. Then

$$
\left|a_{n}^{2}-a_{2 n-1}\right| \leq(n-1)^{2} \quad(n=4,5, \ldots) .
$$

This inequality is sharp.

The proof of the Theorem relies on the following Lemma.

Received by the editors June 9, 1987.

1980 Mathematics Subject Classification (1985 Revision). Primary 30C50, 30C45. 
LEMMA. Let

(4) $F_{n}(x, y)=\left(n^{2}-6 n+1\right) x^{2}-2(n-1)^{2} x y+4(n-1) \sqrt{1-x^{2}} \sqrt{1-y^{2}}+(n-1)^{2} y^{2}$, where $-1 \leq x \leq 1,-1 \leq y \leq 1$ and $n=4,5, \ldots$ Then

$$
F_{n}(x, y) \leq 4(n-1)^{2}-4 n \text {. }
$$

The equality holds if and only if $(x, y)=(1,-1)$ or $(x, y)=(-1,1)$.

PROOF. By applying the inequality $2 a b \leq a^{2}+b^{2}$, we have

$$
F_{n}(x, y) \leq 4(n-1)+G_{n}(x, y)
$$

where

$$
G_{n}(x, y)=\left(n^{2}-8 n+3\right) x^{2}-2(n-1)^{2} x y+\left(n^{2}-4 n+3\right) y^{2} .
$$

If $n \geq 8$, it is clear that

$$
G_{n}(x, y) \leq 4 n^{2}-16 n+8
$$

which implies (5). The equality holds in (8) if and only if $(x, y)=(1,-1)$ or $(x, y)=(-1,1)$, so does the equality in (5) since $1-x^{2}=1-y^{2}$ at $(1,-1)$ and $(-1,1)$.

It is not difficuilt to show that

$$
G_{5}(x, y)=-12 x^{2}-32 x y+8 y^{2}, \quad G_{6}(x, y)=-9 x^{2}-50 x y+15 y^{2},
$$

and

$$
G_{7}(x, y)=-4 x^{2}-72 x y+24 y^{2}
$$

maximize only at $(1,-1)$ and $(-1,1)$. This gives the required result $(5)$ for $n=$ $5,6,7$.

From (4), we get for $-1<x<1$ and $-1<y<1$

$$
\begin{aligned}
& \partial F_{4}(x, y) / \partial x=-14 x-18 y-12 x \sqrt{\left(1-y^{2}\right) /\left(1-x^{2}\right)}, \\
& \partial F_{4}(x, y) / \partial y=-18 x+18 y-12 y \sqrt{\left(1-x^{2}\right) /\left(1-y^{2}\right)} .
\end{aligned}
$$

Both of them vanish only if $(x+y)(7 x-9 y)=0$. For $x=-y$ and $-1<y<1$,

$$
G_{4}(x, y)=-13 x^{2}-18 x y+3 y^{2}=8 y^{2}<8 \text {. }
$$

For $x=9 y / 7$ and $-7 / 9<y<7 / 9$,

$$
G_{4}(x, y)=-2040 y^{2} / 49<8 .
$$

Thus $F_{4}(x, y) \leq 12+G_{4}(x, y)<20$ if $-1<x<1$ and $-1<y<1$. It is easy to check that $F_{4}(x, y)$ maximizes on the boundary of $\{(x, y):-1 \leq x \leq 1,-1 \leq y \leq 1\}$ only at $(1,-1)$ and $(-1,1)$. This completes the proof of our lemma.

PROOF OF THE THEOREM. Since $\left|a_{n}^{2}-a_{2 n-1}\right|$ is invariant under rotations, it is sufficient to prove that

$$
\operatorname{Re}\left(a_{n}^{2}-a_{2 n-1}\right) \leq(n-1)^{2} .
$$

It is well known [3] that $H C$ consists of the functions represented by

$$
f(z)=\int_{R}\left(z-\frac{1}{2}\left(e^{i s}+e^{i t}\right) z^{2}\right)\left(1-e^{i t} z\right)^{-2} d \mu(t, s),
$$


where $\mu$ varies over the set of probability measures on $R=[0,2 \pi] \times[0,2 \pi]$. Thus we have

$$
\begin{aligned}
a_{n} & =\int_{R}\left\{n e^{i(n-1) t}-\frac{1}{2}\left(e^{i s}+e^{i t}\right)(n-1) e^{i(n-2) t}\right\} d \mu \\
& =\frac{1}{2} \int_{R}\left\{(n+1) e^{i(n-1) t}-(n-1) e^{i(s+(n-2) t)}\right\} d \mu
\end{aligned}
$$

and

$$
\begin{aligned}
\operatorname{Re}\left(a_{n}^{2}-a_{2 n-1}\right)= & \frac{1}{4}\left\{\int_{R}[(n+1) \cos (n-1) t-(n-1) \cos (s+(n-2) t)] d \mu\right\}^{2} \\
& -\frac{1}{4}\left\{\int_{R}[(n+1) \sin (n-1) t-(n-1) \sin (s+(n-2) t)] d \mu\right\}^{2} \\
& -\int_{R}[n \cos 2(n-1) t-(n-1) \cos (s+(2 n-3) t)] d \mu .
\end{aligned}
$$

By applying the Cauchy-Schwarz inequality to

$$
\left\{\int_{R}[(n+1) \cos (n-1) t-(n-1) \cos (s+(n-2) t)] d \mu\right\}^{2},
$$

we get

$$
\begin{array}{r}
\operatorname{Re}\left(a_{n}^{2}-a_{2 n-1}\right) \\
\leq \frac{1}{4} \int_{R}\left\{(n+1)^{2} \cos ^{2}(n-1) t\right. \\
-2(n+1)(n-1) \cos (n-1) t \cos (s+(n-2) t) \\
\left.\quad+(n-1)^{2} \cos ^{2}(s+(n-2) t)\right\} d \mu \\
-\int_{R}\{n \cos 2(n-1) t-(-1) \cos (s+(2 n-3) t)\} d \mu \\
\quad n+\frac{1}{4} \int_{R} \quad\left\{\left(n^{2}-6 n+1\right) \cos ^{2}(n-1) t\right. \\
=\quad-2(n-1)^{2} \cos (n-1) t \cos (s+(n-2) t) \\
\quad+(n-1)^{2} \cos { }^{2}(s+(n-2) t) \\
\quad-4(n-1) \sin (n-1) t \sin (s+(n-2) t)\} d \mu \\
\leq n+\frac{1}{4} \int_{R} F_{n}(\cos (n-1) t, \cos (s+(n-2) t)) d \mu .
\end{array}
$$

If follows from the Lemma that

$$
\operatorname{Re}\left(a_{n}^{2}-a_{2 n-1}\right) \leq n+\frac{1}{4} \int_{R}\left(4(n-1)^{2}-4 n\right) d \mu=(n-1)^{2},
$$

and if the equality holds, we must have

$$
\cos (n-1) t=1 \quad \text { and } \quad \cos (s+(n-2) t)=-1
$$

or

$$
\cos (n-1) t=-1 \quad \text { and } \quad \cos (s+(n-2) t)=1
$$


Moreover, the support of $\mu$ is contained in $E_{1}$ or $E_{2}$, where

$$
\begin{gathered}
E_{1}=\{(t, s): t=2 k \pi /(n-1), s=(2 m-1) \pi-2(n-2) k \pi /(n-1), \\
k=0,1, \ldots, n-1, \text { and } m \text { is an integer in } \\
\left.\left[\frac{1}{2}+(n-2) k /(n-1), 3 / 2+(n-2) k /(n-1)\right]\right\}, \\
E_{2}=\{(t, s): t=(2 k+1) \pi /(n-1), s=2 m \pi-(2 k+1)(n-2) \pi /(n-1), \\
k=0,1, \ldots, n-2, \text { and } m \text { is an integer in } \\
\left.\left[\frac{1}{2}(n-2)(2 k+1) /(n-1), 1+\frac{1}{2}(n-2)(2 k+1) /(n-1)\right]\right\} .
\end{gathered}
$$

Because $\sin (n-1) t$ and $\sin (s+(n-2) t)$ vanish on $E_{1}$ and $E_{2},(11)$ is sharp in $H C$, and so is (3). We complete the proof of the Theorem.

It is well known that $S^{*} \subset C \subset H C$. If $f(z) \in C$ with the form (10) and $\mu$ has its support in $E_{i}(i=1,2)$, then the univalence of $f(z)$ implies that $f(z)$ has only one pole of order two on $|z|=1$, from which we can show that

$$
f(z)=\left(z-\frac{1}{2}\left(e^{i s}+e^{i t}\right) z^{2}\right) /\left(1-e^{i t} z\right)^{2},
$$

where $(t, s)$ is in $E_{i}$. Therefore we have the following corollary.

COROLlaRY 1. If $f(z)=z+a_{2} z^{2}+\cdots \in C$, then

$$
\left|a_{n}^{2}-a_{2 n-1}\right| \leq(n-1)^{2} \quad(n=4,5, \ldots) .
$$

The equality holds if and only if $f(z)$ has the form of (12).

Some of the functions with form (12) are in $S^{*}$, so we have the following result given in [1].

COROLlaRY 2. If $f(z)=z+a_{2} z^{2}+\cdots \in S^{*}$, then

$$
\left|a_{n}^{2}-a_{2 n-1}\right| \leq(n-1)^{2} \quad(n=4,5, \ldots) .
$$

This inequality is sharp.

REMARKS. In [1], the authors pointed out that the Zalcman conjecture is false in $H S^{*}$ for $n=2$ and 3 , so it is also false in $H C$ for $n=2$ and 3. For $n=2$, the conjecture is true in $S$, so also in $C$. However, the conjecture remains open in $C$ for $n=3$.

ACKNOWLEDGMent. The author wishes to thank Professor L. A. Zalcman for commenting on his conjecture during his visit to Northwest University.

\section{REFERENCES}

1. J. E. Brown and A. Tsao, On the Zalcman conjecture for starlike and typically real functions, Math. Z. 191 (1986), 467-474.

2. M. Fekete and G. Szegö, Eine Bemerkung über ungerade schlichte Funktionen, J. London Math. Soc. 8 (1933), 85-89.

3. L. Brickman, T. H. MacGregor and D. R. Wilken, Convex hulls of some classical families of univalent functions, Trans. Amer. Math. Soc. 156 (1971), 91-107.

Department of Mathematics, Northwest University, Xian, The People's REPUBLIC OF CHINA

Current address: Department of Mathematical Sciences, University of Cincinnati, Cincinnati, Ohio 45221 